\title{
Innate Imprinting of Murine Resident Alveolar Macrophages by Allergic Bronchial Inflammation Causes a Switch from Hypoinflammatory to Hyperinflammatory Reactivity
}

\author{
Thomas Naessens, ${ }^{*}$ Seppe Vander Beken, ${ }^{\star \dagger}$ \\ Pieter Bogaert, ${ }^{\ddagger}$ Nico Van Rooijen, ${ }^{\S}$ \\ Stefan Lienenklaus, " Siegfried Weiss," \\ Stefaan De Koker, ${ }^{*}$ and Johan Grooten* \\ From the Department of Biomedical Molecular Biology," Ghent \\ University, Ghent, Belgium; the Department of Dermatology and \\ Allergic Diseases, ${ }^{\dagger}$ University of Ulm, Ulm, Germany; the \\ Department of Molecular Biomedical Research, ${ }^{\ddagger}$ Flanders \\ Institute for Biotechnology (VIB), Ghent, Belgium; the \\ Department of Molecular Cell Biology, ${ }^{\circledR}$ University Amsterdam \\ Medical Center, Amsterdam, The Netherlands; and the \\ Laboratory of Molecular Immunology, "Helmboltz Center for \\ Infection Research, Braunschweig, Germany
}

Resident alveolar macrophages (rAMs) residing in the bronchoalveolar lumen of the airways play an important role in limiting excessive inflammatory responses in the respiratory tract. High phagocytic activity along with hyporesponsiveness to inflammatory insults and lack of autonomous IFN- $\beta$ production are crucial assets in this regulatory function. Using a mouse model of asthma, we analyzed the fate of rAMs both during and after allergic bronchial inflammation. Although nearly indistinguishable phenotypically from naïve rAMs, postinflammation rAMs exhibited a strongly reduced basal phagocytic capacity, accompanied by a markedly increased inflammatory reactivity to Toll-like receptors TLR-3 (poly I:C), TLR-4 [lipopolysaccharide (LPS)], and TLR-7 (imiquimod). Importantly, after inflammation, rAMs exhibited a switch from an IFN- $\beta$-defective to an IFN- $\beta$-competent phenotype, thus indicating the occurrence of a new, inflammatory-released $\mathrm{rAM}$ population in the postallergic lung. Analysis of rAM turnover revealed a rapid disappearance of naïve rAMs after the onset of inflammation. This inflammation-induced rAM turnover is critical for the development of the hyperinflammatory rAM phenotype observed after clearance of bronchial inflammation. These data document a novel mechanism of innate imprinting in which noninfectious bronchial inflammation causes alveolar macrophages to acquire a highly modified innate reactivity. The resulting increase in secretion of inflammatory mediators on TLR stimulation implies a role for this phenomenon of innate imprinting in the increased sensitivity of postallergic lungs to inflammatory insults. (Am J Pathol 2012, 181:174-184; bttp://dx. doi.org/10.1016/j.ajpath.2012.03.015)

The mucosal surfaces of the respiratory tract are continuously exposed to environmental antigens and must therefore restrain excessive inflammatory responses in order to fulfill their role in gaseous exchange and to prevent bystander tissue damage. Powerful mechanical and immunosuppressive mechanisms protect the lung against development of inappropriate immune reactivity and inflammation. When these defense mechanisms fail, chronic airway inflammatory diseases such as allergic asthma may develop. ${ }^{1}$ In allergic asthma, the infiltration of the bronchial mucosa by leukocytes (mainly eosinophils), along with subepithelial fibrosis, goblet cell hyperplasia, and airway hyperresponsiveness, leads to reversible loss of lung function and in the long term to irreversible tissue remodeling. ${ }^{2}$

Over several decades, immunosuppressive mechanisms have been identified that inhibit or limit the development of maladaptive pulmonary inflammatory responses. Allergen uptake and presentation by pulmonary

Supported by research funding from the Strategic Basic Research program (SBO; 80016) and the Fund for Scientific Research program (FWO; 3G 006309) from the Flemish government and the Interuniversity Attraction Poles (IAP) 6/18 program from the Belgian federal government. T.N. is a Ph.D. fellow with the Agency for Innovation by Science and Technology (IWT).

Accepted for publication March 22, 2012.

Supplemental material for this article can be found at http://ajp. amjpathol.org or at http://dx.doi.org/10.1016/j.ajpath.2012.03.015.

Address reprint requests to Johan Grooten, Ph.D., Laboratory of Molecular Immunology, Department of Biomedical Molecular Biology, Ghent University, Technologiepark 927, B-9052 Ghent, Belgium. E-mail: johan.grooten@ugent.be. 
plasmacytoid dendritic cells provides intrinsic protection against inflammatory responses to harmless antigen by skewing T-cell differentiation toward the tolerogenic $\mathrm{CD}^{+}{ }^{+} \mathrm{CD}_{25}{ }^{+}$regulatory T-cell phenotype. ${ }^{3}$ Immunomodulatory cytokines such as IL-10 and TGF- $\beta$ are known to possess anti-inflammatory activities in the development of allergic asthma. In addition to their inhibitory effect on proinflammatory cytokine secretion and leukocyte maturation, IL-10 and TGF- $\beta$ are commonly implicated in the generation of the inducible regulatory T-cell subsets $\operatorname{Tr} 1$ and Th3, respectively. ${ }^{4}$ Resident alveolar macrophages (rAMs) are important sources of pulmonary IL-10 and TGF- $\beta,{ }^{5}$ and have been shown to exert an immunosuppressive effect on T-lymphocytes and dendritic cells. ${ }^{6}$ Several studies have demonstrated a significant increase in allergic inflammation and T-cell reactivity in antigen-challenged lungs after depletion of rAMs. ${ }^{7-9}$ The increased sensitivity of rAM-depleted lungs to antigen exposure observed in these studies was attributed to the loss of rAM-mediated suppression of dendritic cell maturation, function, and trafficking to mediastinal lymph nodes. ${ }^{10,11}$ Along with the mucosal epithelia, rAMs are the first cells to contact inhaled infectious agents, as well as small-particulate debris and airborne allergens.

After resolution of inflammation, the increased numbers and altered differentiation of long-lived antigen-reactive lymphocytes, a hallmark of the adaptive immune branch, stand in strong contrast to the return to basal levels for cell numbers and differentiation that are commonly reported for innate immune cells such as tissue macrophages. Recent reports, however, increasingly challenge the paradigm of innate cells retaining no memory of prior inflammatory insults. ${ }^{12}$ Murine rAMs have been reported to display a sustained desensitization to bacterial Toll-like receptor (TLR)-5 ligands after resolution of respiratory influenza infection. ${ }^{13}$ In a mouse model of Sendai virus infection, a lasting effect on rAMs was observed, one that persisted after clearance of the virus. In this model of paramyxovirus-induced pathology, rAM activation persisted, resulting in a chronic lung condition with pathological features resembling asthma and chronic obstructive pulmonary disease. ${ }^{14}$ These observations indicate that infection may educate innate immune cells also, altering the way the cells respond to a subsequent inflammatory insult. This concept of innate imprinting has been documented in several mouse models of infection. ${ }^{12}$ Nonetheless, the extent to which innate imprinting also occurs after noninfectious, allergic inflammation and the nature of its functional outcome remain largely unknown.

Here, we now provide evidence for a pronounced innate imprinting of rAMs as a consequence of allergic bronchial inflammation in mouse models for eosinophilic, Th2-biased mild to moderate asthma and for neutrophilic, Th1- and Th17-biased severe refractory asthma. The altered functional maturation of postinflammatory rAMs was evidenced by an enhanced responsiveness of the cells to TLR ligands and a newly acquired capacity to produce IFN- $\beta$, a type I IFN. Mechanistically, we provide evidence that the switch from a restrained to an unrestrained rAM inflammatory response is the consequence of allergic inflammation-induced rAM turnover, accompanied by the emergence after clearance of inflammation of a new population of secondary rAMs with increased reactivity to inflammatory insults.

\section{Materials and Methods}

\section{Mice}

Female C57BL/6 mice, 6 to 8 weeks old, were purchased from Janvier (Le Genest-St-Isle, France). This wild-type strain expresses the CD45.2 alloantigen and served as recipient for the generation of $\mathrm{CD} 45$ chimeric mice. Female B6.SJL-Ptprca Pep3b/BoyJ mice, 12 weeks old, were purchased from Charles River International (Brussels, Belgium). This strain expresses the CD45.1 alloantigen and served as bone marrow donor for the generation of CD45 chimeric mice. Both mice strains were kept under specific pathogen-free conditions. All animal experiments were approved by the local ethics committee.

\section{Mouse Models of Allergic Airway Inflammation}

For the allergic asthma model, C57BL/6 mice were immunized intraperitoneally with $20 \mu \mathrm{g}$ of grade $\mathrm{V}$ chicken egg ovalbumin (OVA; Sigma-Aldrich, St. Louis, MO), adsorbed on $1 \mathrm{mg}$ aluminum hydroxide (alum; Sigma-Aldrich) in endotoxin-free PBS (Lonza, Walkersville, MD). To generate a model for a noneosinophilic severe refractory Th1-Th17mediated allergic bronchial inflammation, C57BL/6 mice were immunized subcutaneously with $20 \mu \mathrm{g}$ of OVA in PBS emulsified in $75 \mu \mathrm{L}$ complete Freund's adjuvant (CFA; SigmaAldrich). In both mouse models, OVA-sensitized mice were exposed to OVA aerosols, consisting of either 1\% (allergic asthma model) or $0.1 \%$ (neutrophilic Th1-/Th17-mediated allergic inflammation model) of grade III OVA in PBS.

\section{Generation of Chimeric CD45.2 Alloantigen- Expressing Recipient Mice}

Bone marrow cells were isolated under sterile conditions from the tibias and femurs of sex-matched CD45.1 donor mice. Briefly, tibias and femurs were flushed with sterile PBS and the cell suspension was filtered through 70- $\mu \mathrm{m}$ nylon mesh (BD Biosciences, San Diego, CA) to remove cell aggregates. Red blood cell lysis was performed before transplantation by incubation of the single-cell suspension in ACK lysing buffer (Lonza) for 3 minutes at room temperature. Recipient CD45.2 alloantigen-expressing C57BL/6 mice received 8 Gy of total body irradiation using a 5-MV photon beam of a linear accelerator (SL-75, Elekta, Crawley, UK). This radiation dose nearly completely depleted the bone marrow, but did not induce depletion of rAMs and memory T cells in previously OVAalum-sensitized mice. A total of $8 \times 10^{6} \mathrm{CD} 45.1$ donor bone marrow cells suspended in $250 \mu \mathrm{L}$ sterile PBS were transplanted via lateral tail-vein injections into CD45.2 recipient mice. The drinking water of the $\mathrm{CD} 45.2$ recipient mice was supplemented with $0.2 \%$ neomycin trisulfate antibiotics (Sigma-Aldrich) from 5 days before until 14 days after the irradiation. 


\section{Alveolar Cell Isolation and Culture}

Mice were anesthetized with 2,2,2-tribromomethanol (Avertin; Sigma-Aldrich), 2,5\% in PBS. Bronchoalveolar lavage (BAL) was performed by making a small incision in the trachea, to allow passage of a lavage cannula. Lungs were flushed four times with $1 \mathrm{~mL} \mathrm{Ca}^{2+}$-free and $\mathrm{Mg}^{2+}$-free Hank's balanced salt solution (HBSS; Invitrogen Life Technologies, Carlsbad, CA), supplemented with $0.05 \mathrm{mmol} / \mathrm{L}$ EDTA (ethylenediaminetetraacetic acid). Optionally, a prior lavage with $0.5 \mathrm{~mL}$ HBSS-EDTA was performed and BAL fluid (BALF) was isolated by centrifugation and collection of the supernatant. BALF cells were washed and resuspended in PBS for further use. Pre- and postinflammation rAMs isolated via BAL were cultured in complete culture medium: RPMI 1640 medium containing $1 \%$ heat-inactivated fetal calf serum, $25 \mathrm{mmol} / \mathrm{L}$ HEPES, $2 \mathrm{mmol} / \mathrm{L}$ L-glutamine, $1 \mathrm{mmol} / \mathrm{L}$ pyruvate, $100 \mathrm{U} / \mathrm{mL}$ penicillin/streptomycin (Invitrogen-Life Technologies), and $55 \mu \mathrm{mol} / \mathrm{L}$ 2-mercaptoethanol (SigmaAldrich). All cultures were enriched for macrophages by plastic adhesion for 1 hour at $37^{\circ} \mathrm{C}$. Naïve and postinflammation rAMs were then stimulated for the indicated times with LPS (Escherichia coli 0111:B4; Sigma-Aldrich), polyriboinosinic:polyribocytidylic acid (poly I:C; InvivoGen, San Diego, CA), or imiquimod (InvivoGen) at $37^{\circ} \mathrm{C}$.

\section{Flow Cytometry}

The expression of alveolar macrophage maturation markers was assessed on naïve and postinflammation rAMs by flow cytometry. Briefly, BALF cells were counted and suspended at a concentration of $10^{6}$ cells $/ \mathrm{mL}$. High-affinity $F_{c} \gamma$ receptors (Fc $\gamma R s$ ) were blocked by incubation with purified anti-mouse CD16/CD32 (Fc Block) for 15 minutes at $4^{\circ} \mathrm{C}$ and were stained with $\mathrm{CD} 11 \mathrm{c}$-allophycocyanin (APC), DEC-205-phycoerythrin (PE), F4/80-biotin, CD11b-PE, and/or CD115-PE for 1 hour at $4^{\circ} \mathrm{C}$. Biotinylated $\mathrm{F} 4 / 80$ antibody was detected by an additional incubation step with streptavidin-PE for 20 minutes at $4^{\circ} \mathrm{C}$. All antibodies and the streptavidin-PE were purchased from BD Biosciences. Autofluorescence was detected in the FL-1 channel.

Turnover of naïve rAMs during the course of the bronchial inflammation elicited in both models was determined using CD45 chimeric mice. Naïve rAMs were identified by recipient-specific CD45.2 expression and by uptake of Fluoresbrite Yellow Green (YG) plain 1- $\mu$ m latex microspheres (Polysciences, Warrington, PA), administered by the intrathecal route 48 hours before the first OVA aerosol exposure. Elicited cells were identified as microsphere $^{-}$cells expressing donor CD45.1. Antimouse CD45.1-PE and CD45.2-PerCP-Cy5.5 antibodies (BD Biosciences) were used according to the manufacturer's instructions. Preincubation of the cells with $\mathrm{FC}_{\mathrm{C}}$ Block was used to prevent unwanted binding to Fc receptors. All samples were measured on a FACSCalibur flow cytometer (BD Biosciences) and were analyzed using CellQuest Pro software version 6.0 (BD Biosciences).

\section{BALF Total and Differential Cell Counts}

BALF cell counts and cell-type composition was analyzed by flow cytometry. Cells preincubated with Fc Block were classified as monocytes (alveolar macrophages, elicited monocytes, and dendritic cells), neutrophils, eosinophils, or lymphocytes based on forward and side scatter gating and fluorescence intensities for anti-mouse CD3 $\varepsilon$-Alexa488, B220-FITC, CCR3-PE, CD11C-APC, and $\mathrm{I}-\mathrm{A}^{\mathrm{b}}$-biotin that was recognized by streptavidin-PerCP. All antibodies and streptavidin-PerCP were from BD Biosciences, except that CCR3-PE was from R\&D Systems (Abingdon, UK). Additionally, the total number of BALF cells was calculated from the measured total cell count relative to the number of Flow-Count beads (Beckman Coulter, Brea, $\mathrm{CA}$ ), of which a constant amount of was added to the sample. Total numbers of BALF cells were counted by use of a Bürker chamber (Marienfeld, Lauda-Königshofen, Germany). Trypan Blue was added to exclude dead cells. Differential cell counts obtained by flow cytometry were confirmed by morphological examination of cytospin preparations using a Shandon cytocentrifuge (TechGen, Zellik, Belgium) and stained with May-Grünwald-Giemsa stain (Sigma-Aldrich). The percentage of monocytes or macrophages, neutrophils, and eosinophils was determined by counting at least 400 cells. Both analyses were performed on an Olympus BX51 microscope (Olympus, Tokyo, Japan) equipped with $4 \times, 10 \times, 20 \times, 40 \times$, and $100 \times$ objectives.

\section{Total RNA Preparation and Real-Time Quantitative PCR}

RNA isolation was performed using an RNeasy Plus mini kit (Qiagen, Hilden, Germany) according to manufacturer's protocol. cDNA was synthesized using a SuperScript II reverse transcription reagent kit (Invitrogen-Life Technologies). Real-time quantitative PCR (qPCR) was performed on a LightCycler 480 system using a qPCR kit for SYBR Green I (both from Roche Molecular Systems, Pleasanton, CA). Real-time qPCR amplification was performed in triplicate reactions under the following conditions: a preincubation step at $95^{\circ} \mathrm{C}$ for 5 minutes, followed by 50 cycles at $95^{\circ} \mathrm{C}$ for 10 seconds and at $60^{\circ} \mathrm{C}$ for 30 seconds. The following primers were used, forward and reverse, respectively: murine Arg1, 5'-TGAACACGGCAGTGGCTTA-3' and 5'-GCATTCACAGTCACTTAGGTGGTTTA-3'; murine Inos, 5'CAGCTGGGCTGTACAAACCT-3' and 5'-CATTGGAAGTGAAGCGTTTCG-3'; murine Usp18, 5'-AGCCCTCATGGTCTGGTTGGTT-3' and 5'-GCACTCCGAGGCACTGTTATCC-3'; murine Ifit2, 5'-ATCTCTCCCTACTCTGCCCTCCTA-3' and 5'-GCGTATAAATCAGCAATCCCTTCA-3'; murine Oas1 (Oas1a form), 5' -CCCTGGGCCCTTCCTGT-3' and 5' -CCCGGGGGCACTTGTCT-3'; murine Ifi205, 5' -GGATAGAAGTTGTGGGGAGTGGC-3' and 5'-CAGCCTTGGTGACCTTGACGA-3'; murine $\| 6$, 5'-TAGTCCTTCCTACCCCATTTCC-3' and 5'-TTGGTCCTTAGCCACTCCTTC3'; murine //12p35), 5'-AACCAGGGCCTTCTTTAG-3' and 5'-GATCTGCCTGCCTTGGTCT-3'; and murine Rp/13a, 5'-CCTGCTGCTCTCAAGGTTGTT-3' and 5'-TGGCTGTCACTGCCTGGTACTT-3'. Murine RPL13a mRNA was used as reference housekeeping gene for normalization. 
All primers were purchased from Invitrogen-Life Technologies.

\section{In Vitro Phagocytosis Assay}

Uptake of $\mathrm{YG}^{+}$microspheres by naïve and postinflammation rAMs was imaged with a confocal microscope (TCS SP5 system with an acousto-optical beam splitter; Leica, Wetzlar, Germany) using a 488-nm multiple argon laser line. Cytoplasm was stained with CellTracker Orange (Invitrogen-Life Technologies) and was excited with a 543-nm helium-neon laser. Nuclei were stained with $500 \mathrm{nmol} / \mathrm{L}$ DAPI (Invitrogen-Life Technologies) and excited with the 405-nm line of an UV diode laser. Stained cells were mounted in 1\% N-propyl gallate in glycerol before image acquisition. Images were acquired using LAS AF software version 2.4.1, build 6384 (Leica) and were subsequently analyzed with Volocity software version 5.5 (PerkinElmer, Coventry, UK).

\section{Cytokine and Chemokine Measurement}

Protein levels of mouse TNF- $\alpha$, IL-6, IL-12p70, CXCL1, and CXCL2 in culture supernatant or BALF were quantified with a Bio-Plex suspension array system (Bio-Rad Laboratories, Hercules, CA) for simultaneous detection of cytokines, according to the manufacturer's protocol. The analytes were measured with a Bio-Plex protein array reader and Bio-Plex manager software version 5.0 (Bio-Rad Laboratories), using recombinant cytokine standards (all from Bio-Rad Laboratories). Culture supernatant levels of IFN- $\beta$ were determined using a VeriKine mouse IFN- $\beta$ ELISA kit (PBL Interferon Source, Piscataway, NJ) according to the manufacturer's protocol.

\section{Statistical Analysis}

Statistical analyses were performed using GraphPad Prism software version 5 (GraphPad Software, La Jolla, CA). Outlier statistics were used to choose between performing a one-way analysis of variance or Kruskal-Wallis nonparametric test, and Gaussian distribution of parameters was checked using a Kolmogorov-Smirnov test. Differences in means between each of two independent experimental groups were analyzed using an unpaired $t$-test or the nonparametric Mann-Whitney U-test at the 95\% confidence interval. No statistical analysis was done for gene expression data, because these data involved pooled samples.

\section{Results}

\section{Surface Marker Profile and Basal Differentiation of Pre- and Postinflammation rAMs}

We used a mouse model of allergic asthma in which a Th2-biased sensitization of C57BL/6 mice against the model allergen OVA is elicited by repeated intraperitoneal immunization using aluminum hydroxide as an adjuvant. ${ }^{3}$ Exposure of the sensitized mice to nebulized OVA then generated an eosinophilic airway inflammation
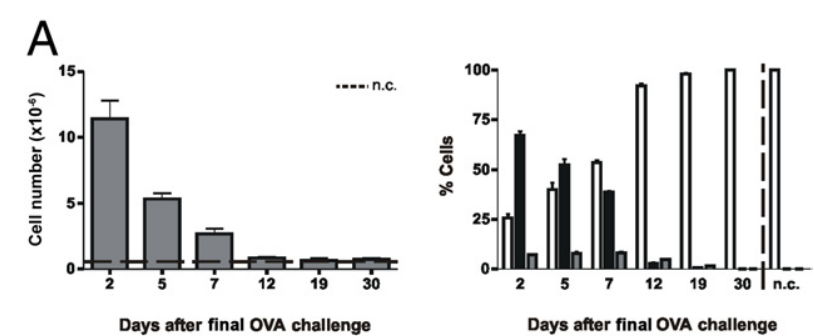

B
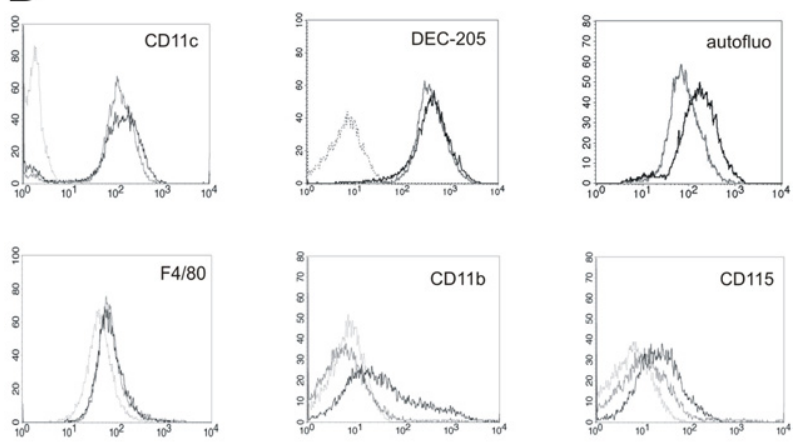

C
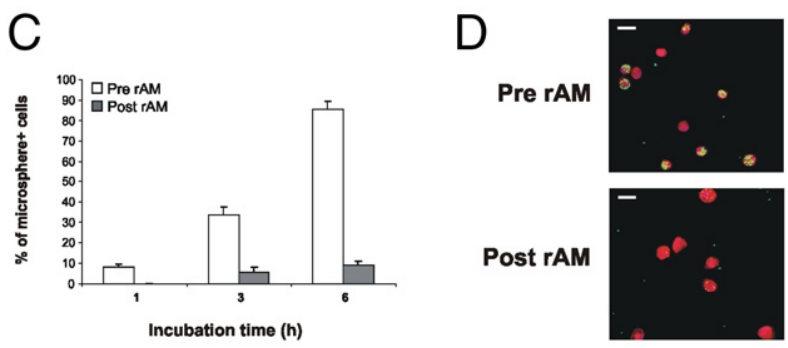

E

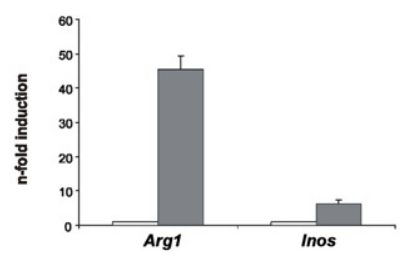

Figure 1. Marker profile and basal differentiation of postinflammation rAMs. A: OVA-alum-sensitized C57BL/6 mice were exposed to seven OVA challenges or were left untreated as naïve controls (n.c.). Average total cell counts (left) and differential cellular composition (right) cell counts for BALF were determined via flow cytometry and Giemsa and MayGrünwald staining cytospin analyses, respectively, at 2 to 30 days after the final OVA exposure $(n=5)$. Differential: white, macrophages; black, neutrophils; gray, eosinophils. Error bars indicate SEM. B: Flow cytometry analysis of the expression pattern of alveolar macrophage maturation markers. OVA-alum-sensitized C57BL/6 mice were exposed seven times OVA challenges $(n=7)$ or were left untreated $(n=7)$. BALF samples were taken at day 15 after the final OVA exposure, and naïve (gray) and postinflammation (black) rAMs were analyzed for intrinsic fluorescence intensity and for expression of CD11c, DEC-205, F4/80, CD11b, and CD115. Dotted line, unstained control. C and D: Pre- and postinflammation rAMs isolated from $\mathrm{C} 57 \mathrm{BL} / 6$ mice $(n=5)$ were incubated for 1,3 , or 6 hours with $\mathrm{YG}^{+}$microspheres $(10$ microspheres/cell). Microsphere uptake was determined by confocal analysis of the percentage of microsphere $^{+}$cells $(\mathbf{C})$ and the number of microspheres per cell $(\mathbf{D})$. The percentage of microsphere ${ }^{+}$rAMs represents the average of 10 randomly selected microscopic fields from triplicate cultures. Error bars indicate SEM. Confocal images in $\mathbf{D}$ are an overlay of green (YG microsphere), red (CellTracker Orange), and blue (DAPI nuclear staining). Scale bar $=20$ $\mu \mathrm{m}$. E: Pre- and postinflammation rAMs were isolated $(n=7)$, and the basal levels of Arg-1 and Inos mRNA transcripts were assessed by qPCR. Data (E) are expressed as means \pm SD and are representative of two independent experiments; $n$-fold induction is relative to naïve rAMs from triplicate PCR reactions. 
mimicking the immunopathology of mild to moderate asthma (Figure 1A). The clearance of the allergic pulmonary inflammation was verified by harvesting BALF samples at different time points after the last of seven OVA challenges. This showed that the alveoli regained a new steady-state condition within 12 days. At this time point, absolute cell numbers had returned to basal levels (Figure $1 \mathrm{~A}$ ), and cytospin analysis showed that the cellular composition of the alveoli again was at 90\% macrophages (Figure 1A). These macrophages form the new rAM population of postinflammation lungs and can therefore be considered postinflammation rAMs. In addition, at this time point Th2-associated inflammatory cytokines were no longer detectable in the BALF (data not shown).

To determine to what extent the postinflammation rAM population exhibited a characteristic alveolar macrophage phenotype, we analyzed expression levels of alveolar macrophage markers by flow cytometry. Naïve and postinflammation rAM populations were isolated via BAL from naïve mice and OVA-challenged mice 15 days after the final OVA exposure. CD11C and DEC-205, hallmark surface markers of $\mathrm{rAMs},{ }^{15-20}$ were equally and uniformly expressed on both rAM populations (Figure 1B). High intrinsic fluorescence intensity, which is a general phenotypic characteristic of $\mathrm{rAMs},{ }^{21}$ was present in both rAM populations. Also, F4/80, a broad macrophage marker, ${ }^{22}$ was nearly equally and uniformly expressed on both cell populations. In contrast, naïve rAMs were uniformly negative for the expression of CD11b, whereas postinflammation rAMs expressed medium to high levels of CD11b. Furthermore, the monocyte marker CD115 (MCSFR) and phagocytosis receptors Fc $\gamma$ RIII (CD16/CD32) and SR-A (CD36) exhibited uniformly elevated expression levels at rAMs from postinflammation mice (Figure 1B; see also Supplemental Figure S1 at http://ajp. amjpathol.org). Thus, although postinflammation rAMs exhibit a characteristic alveolar macrophage marker profile (ie, autofluo ${ }^{\text {high }} \mathrm{CD}_{11 \mathrm{C}^{+}} \mathrm{DEC} 205^{+} \mathrm{F} 4 / 80^{+}$), they differ from naïve rAMs in the expression levels of macrophage/ monocyte maturation and phagocytosis markers.

Alveolar macrophages typically exhibit high phagocytic activity. ${ }^{23}$ To verify to what extent postinflammation rAMs had retained this functional trait, naïve and postinflammation rAMs were incubated ex vivo with fluorescent latex microspheres for up to 6 hours. Phagocytosis of the latex microspheres was assessed by confocal microscopy. The microspheres were readily engulfed by naïve rAMs, whereas microsphere uptake was strongly reduced in postinflammation rAMs at all time points assayed (Figure 1C). After 6 hours of incubation, nearly $85 \%$ of naïve rAMs were positive for uptake of microspheres, compared with only $10 \%$ of postinflammation rAMs. In addition, microsphere $^{+}$naïve rAMs consistently featured higher numbers of microspheres per cell, compared with microsphere ${ }^{+}$ postinflammation rAMs (Figure 1D).

Reduced phagocytic activity is often observed in macrophages that have been alternatively differentiated. ${ }^{24}$ These so-called M2 macrophages feature, in addition to a low phagocytic capacity, an arginine metabolism differing from M1 or classical differentiated macrophages by an increased arginase/iNOS expression ratio. ${ }^{25}$ Anal- ysis of Arg1 and Inos mRNA levels indeed confirmed this shift toward an M2 characteristic arginine metabolism. The expression of $\mathrm{Arg}-1$, the prototypic M2 marker, was up to 50-fold higher in postinflammation rAMs, compared with naîve rAMs, whereas the expression of the M1 marker, Inos, scarcely differed between the two macrophage populations (Figure 1E).

\section{Differential Inflammatory Cytokine and IFN- $\beta$ Response after TLR Stimulation}

Next, to investigate to what extent TLR signaling in postinflammation rAMs is affected, we compared the response of pre- and postinflammation rAMs to ligation of the antibacterial Toll-like receptor TLR- 4 and the antiviral Toll-like receptors TLR-3 and TLR-7. rAMs were isolated from naïve and postinflammation lungs and cultured ex vivo for 6 hours in the presence of E. coli LPS $(0.1 \mu \mathrm{g} / \mathrm{mL})$, poly I:C $(10 \mu \mathrm{g} / \mathrm{mL})$, or imiquimod $(10 \mu \mathrm{g} / \mathrm{mL})$. Production levels of inflammatory cytokines and chemokines were subsequently analyzed via a Bio-Plex suspension array system. Compared with naïve rAMs, postinflammation rAMs exhibited markedly increased protein levels for TNF- $\alpha$, IL-6, IL12p70, CXCL1 (KC), and CXCL2 (MIP-2) after stimulation with LPS and imiquimod (Figure 2). With poly I:C stimulation, however, these cytokines and chemokines remained undetectable or near basal levels (CXCL2) in the supernatant of both rAM cell cultures (Figure 2).

rAMs differ from other tissue macrophages in their failure to autonomously produce IFN- $\beta$ in response to TLR-3 and TLR-4 triggering. ${ }^{26}$ Strikingly, postinflammation rAMs switched from an IFN- $\beta$ production-defective to an IFN- $\beta$ production-competent phenotype after LPS and poly I:C stimulation, but failed to do so in response to imiquimod (Figure 3A). This difference was also confirmed at the level of autocrine IFN- $\beta$ bioactivity, as apparent from the strongly increased transcript levels of Usp18, Ifit2, Oas1, and Ifi205 in LPS- and poly I:C-treated postinflammation rAMs (Figure 3B). The IFN- $\beta$ biomarker function of these genes was confirmed by performing a similar analysis on the postinflammation rAMs from Ifnb $\mathrm{KO}$ mice, ${ }^{27}$ which developed a normal Th2-mediated eosinophilic inflammation (data not shown). Postinflammation rAMs of these $\mathrm{KO}$ mice no longer displayed the strong induction of Usp18, Ifit2, Oas1, and Ifi205 in response to LPS (data not shown), thus confirming the dependence of the genes on the expression of IFN- $\beta$.

\section{Increased in Vivo Inflammatory TLR Reactivity of Postinflammation Lungs}

The functional outcome of the differential TLR reactivity observed between both rAM populations was further verified by analysis of the inflammatory BALF infiltrate 16 hours after instillation of LPS (10 ng), poly I:C (800 ng), or imiquimod $(800 \mathrm{ng})$. In contrast to the relatively weak response observed in naïve lungs, inflammation-experienced lungs exhibited a more pronounced alveolar infiltration of inflammatory leukocytes after challenge with LPS, poly I:C, or imiquimod (Figure 4A). Although macro- 
TNF- $\alpha$

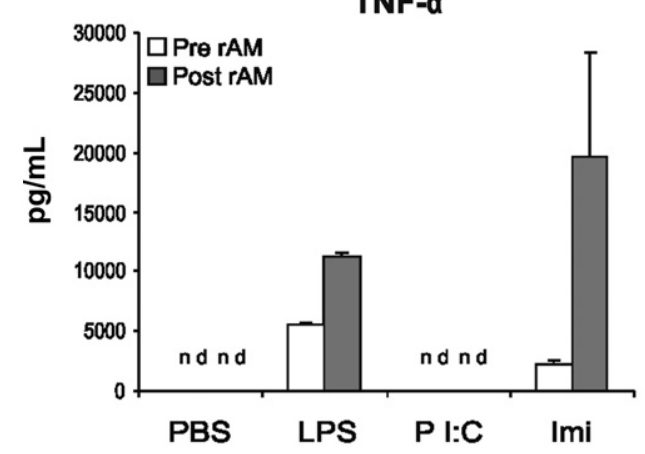

IL-12p70
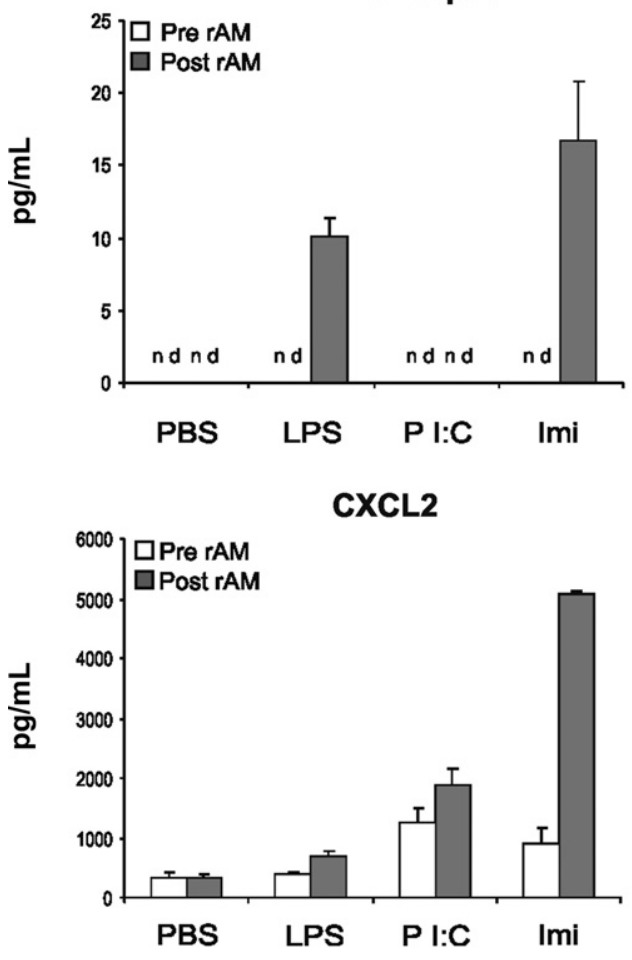

phages/monocytes and neutrophils constituted the main components of the inflammatory infiltrate in both groups, higher cell numbers of both leukocyte populations were found in the BALF isolated from LPS-challenged postinflammation lungs, compared with naïve lungs (Figure 4B). Furthermore, in naivve lungs the instillation of poly $\mathrm{I}: \mathrm{C}$ or imiquimod resulted in an alveolar infiltration of mainly monocytes/macrophages. In contrast, in postinflammation lungs the poly I:C or imiquimod challenge caused in addition a significant recruitment of neutrophils and eosinophils to the bronchoalveolar lumen (Figure 4B).

\section{Inflammation-Induced rAM Turnover Is Crucial for the Development of the Postinflammation rAM Phenotype}

We next addressed the question to what extent the observed postinflammation rAM phenotype arose either from naïve rAMs that underwent an education process
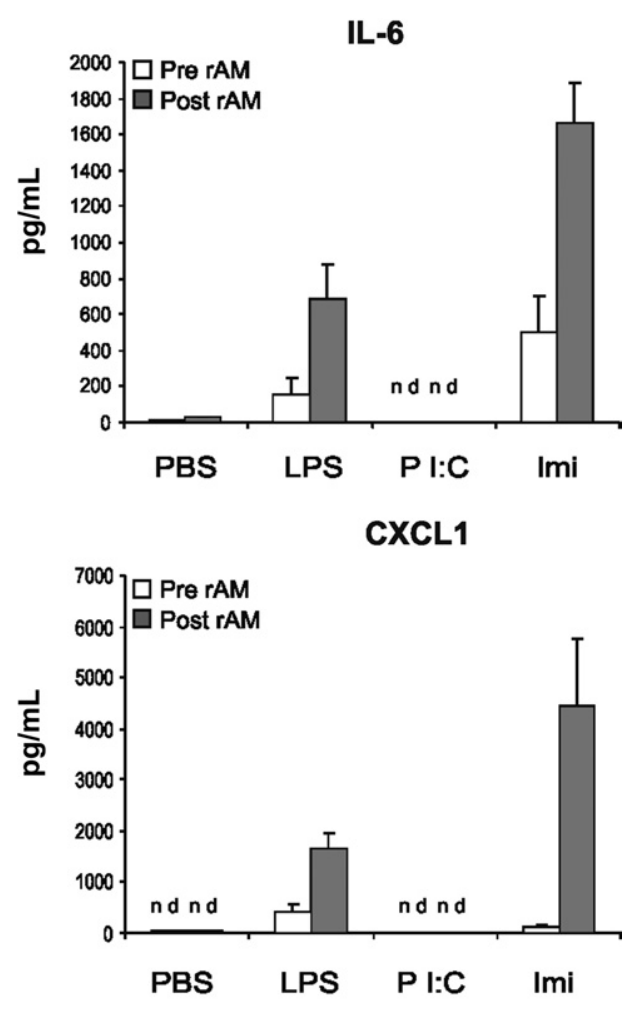

Figure 2. Inflammatory cytokine and chemokine response of pre- and postinflammation rAMs to TLR-3, TLR-4, and TLR-7 engagement. Pre- and postinflammation rAMs isolated from $\mathrm{C} 57 \mathrm{BL} / 6$ mice $(n=8)$ were stimulated ex vivo with $0.1 \mu \mathrm{g} / \mathrm{mL}$ E. coli $\mathrm{LPS}, 10 \mu \mathrm{g} / \mathrm{mL}$ poly I:C (P $\mathrm{I}: \mathrm{C}$ ), or $10 \mu \mathrm{g} / \mathrm{mL}$ imiquimod (Imi) for 6 hours or were left untreated as control. Protein levels of TNF- $\alpha$, IL- 6 , IL12p70, CXCL-1, and CXCL-2 in the culture supernatant were measured using a Bio-Plex suspension array system. Data are expressed as means \pm SD of triplicate culture conditions and are representative of three independent experiments. nd, not detectable.

during pulmonary inflammation or from monocytes that were freshly recruited during or after bronchial inflammation and whose differentiation was subsequently influenced by the prevailing lung environment. We therefore monitored the fate of the naïve rAM population in the course of the allergic bronchial inflammation. To facilitate discrimination between $\mathrm{rAMs}$ and newly recruited macrophages/monocytes, OVA-alum-sensitized C57BL/6 mice, which express the CD45.2 leukocyte alloantigen, were irradiated and then transplanted with CD45.1 alloantigen-expressing bone marrow cells (Figure 5A). In combination with intratracheal delivery of fluorescent yellow-green YG latex microspheres (1 $\mu \mathrm{m})$, flow cytometry analysis allowed identification of rAMs as microsphere ${ }^{+}$ CD $45.2^{+}$cells and recruited macrophages/monocytes as microsphere ${ }^{-}$CD45. $1^{+}$cells (Figure 5B). At the moment of intratracheal delivery of $\mathrm{YG}^{+}$microspheres, a small fraction of newly recruited macrophages from the donor type are present and have taken up the instilled microspheres. This cell fraction accounts for $2 \%$ in the 
A

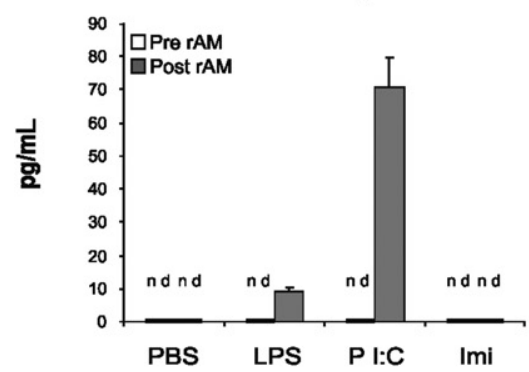

Figure 3. IFN- $\beta$ response of pre- and postinflammation rAMs to TLR-3, TLR- 4 , and TLR-7 engagement. Naïve and postinflammation rAMs were isolated from C57BL/6 mice $(n=8)$ and were stimulated ex vivo with $0.1 \mu \mathrm{g} / \mathrm{mL}$ E. coli LPS, $10 \mu \mathrm{g} / \mathrm{mL}$ poly I:C (P I:C), or $10 \mu \mathrm{g} / \mathrm{mL}$ imiquimod (Imi) for 6 hours or were left untreated as control. A: IFN- $\beta$ protein levels in the culture supernatant were determined by ELISA. Data are expressed as means $\pm \mathrm{SD}$ of triplicate culture conditions. nd, not detectable. B: qPCR was used to analyze mRNA expression levels of Usp18, Ifit2, Oas1 and Ifi205, compared with unstimulated naive rAMs Data are expressed as means \pm SD from triplicate PCR reactions and are representative of three independent experiments; $n$-fold induction is relative to unstimulated naïve rAMs.
B Usp18

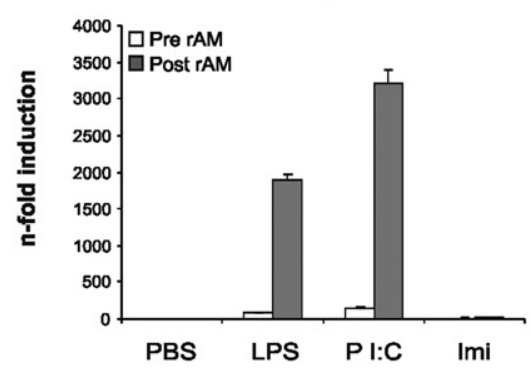

Oas1

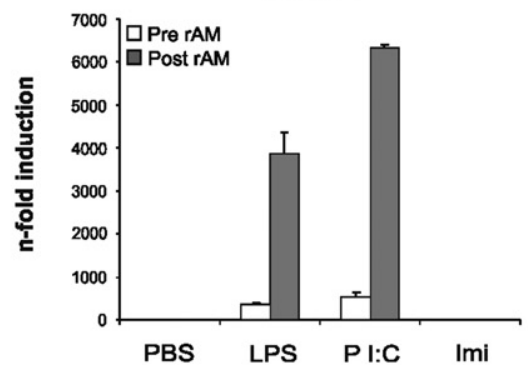

Ifit2

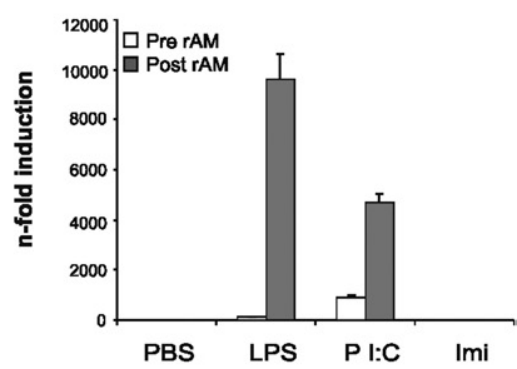

Ifi205

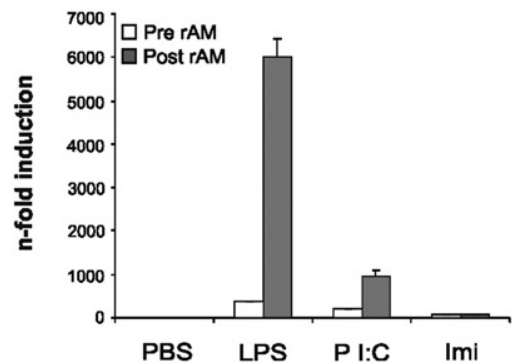

naïve condition (Figure 5B). The pulmonary response to nebulized OVA was not influenced by the preceding irradiation process (see Supplemental Figure S2 at http:// ajp.amjpathol.org). Furthermore, intratracheal administration of microspheres did not elicit inflammatory cell recruitment as such and did not alter the expression of typical alveolar macrophage markers (CD11c, DEC-205, and F4/80) (data not shown).

Exposure to OVA aerosol provoked pronounced inflammatory cell recruitment to the bronchoalveolar lumen (Figure 5B). As a consequence, the relative number of the microsphere ${ }^{+} \mathrm{CD}_{45.2^{+}}$rAMs decreased with increasing numbers of OVA challenges. To determine the inflammation-induced $\mathrm{rAM}$ turnover, we therefore calculated the absolute numbers of microsphere ${ }^{+}$CD45.2 ${ }^{+}$ cells present in the BALF from the percentage of the cell population and the total alveolar cell infiltrate. This analysis revealed that absolute $\mathrm{AAM}$ numbers remained constant after two OVA exposures (Figure 5C); however, microsphere ${ }^{+}$CD45.2 $2^{+}$numbers dropped sharply after four OVA exposures and nearly completely disappeared after seven OVA exposures (Figure 5C). To determine whether fewer OVA exposures would also lead to rAM disappearance from the alveoli, OVA-alum-sensitized CD45 chimeric mice were exposed only twice to OVA aerosol challenge. As expected, absolute rAM numbers remained constant for the first 2 days of treatment, but then decreased dramatically from day 3 after the final (second) OVA challenge (Figure 5D).

We next verified to what extent the phenotype of the postinflammation rAMs was a consequence of the preceding allergic inflammation per se or of the accompanying rAM turnover. We therefore mimicked the turnover of enhanced rAMs and their replacement by newly recruited macrophages/monocytes by a sterile, noninflammatory depletion of naïve rAMs via intrathecally adminis- tered clodronate liposomes. ${ }^{28}$ This depletion was followed by a spontaneous gradual reconstitution of the rAM population, which reached basal levels at day 14 (data not shown). Strikingly, when stimulated ex vivo, this depletion-induced secondary rAM population exhibited a LPS hyporesponsive phenotype highly similar to the hyporesponsiveness of naïve rAMs. No significant differences in mRNA expression levels of the inflammatory cytokine and chemokine genes $/ 16$ and $\| 12$ p35 were found between LPS-stimulated naïve rAMs and depletion-induced secondary rAMs (Figure 6A). The expression of the IFN- $\beta$ responsive genes Usp18 and Ifit2 likewise did not differ between the two rAM populations (Figure 6A).

This dependence of the altered postinflammation rAM reactivity on inflammation-induced $\mathrm{rAM}$ turnover, rather than on rAM turnover per se, raised the issue of the extent to which the nature of the bronchial allergic inflammation may affect this phenomenon of innate imprinting. We therefore applied the same experimental setup, but now in a mouse model of noneosinophilic severe refractory asthma. In this model, systemic sensitization against OVA in the presence of CFA leads to a Th1- and Th17-biased neutrophilic bronchial inflammation after exposure to nebulized OVA. ${ }^{29}$ Also in this model, bronchial inflammation resulted in a drastic decrease in absolute microsphere $^{+}$CD45.2 $2^{+}$rAM numbers after four OVA challenges (Figure 6B), similar to the rAM turnover observed in the course of Th2-biased eosinophilic bronchial inflammation. When assayed for reactivity to LPS-stimulation, 15 days after the final OVA exposure, postinflammation rAMs exhibited the enhanced inflammatory reactivity characteristic of postinflammation rAMs from the eosinophilic asthma model (ie, increased expression levels of $\mathrm{NF}-\kappa \mathrm{B}$ and IFN- $\beta$ responsive genes after ex vivo stimulation with LPS) (Figure 6A). 

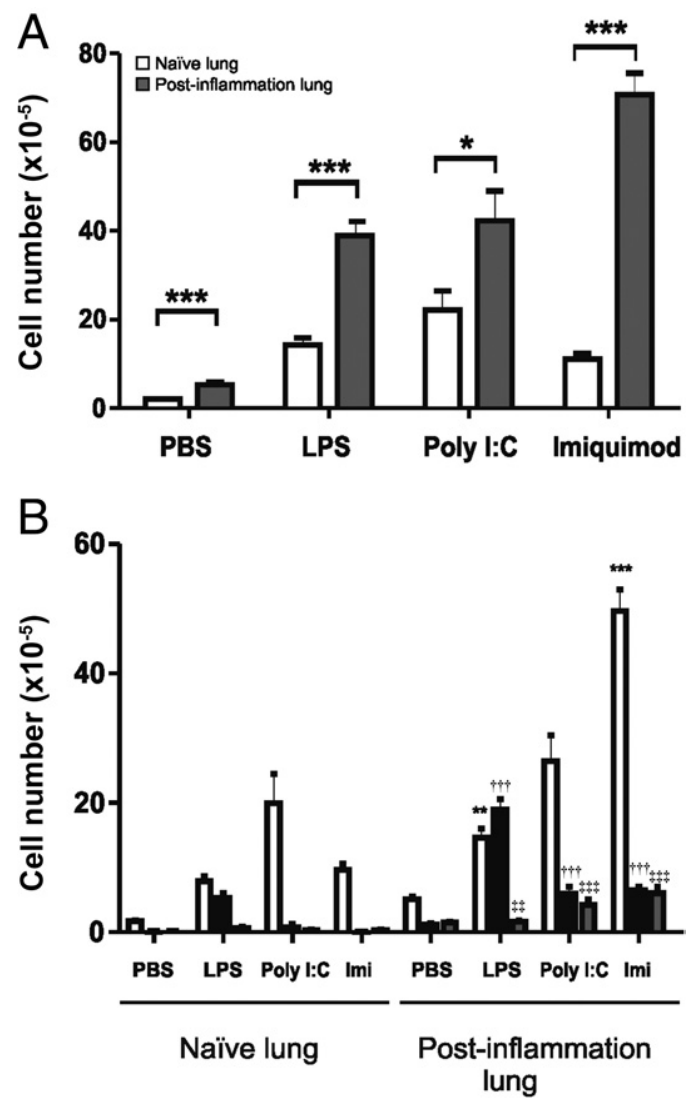

Figure 4. In vivo LPS, poly I:C, and imiquimod challenge of postinflammation lungs. A and B: Postinflammation (day 15 after the final OVA challenge) and naïve $\mathrm{C} 57 \mathrm{BL} / 6$ mice $(n=7)$ were challenged intrathecally with $10 \mathrm{ng} E$. col $i$ LPS, 800 ng poly I:C, 800 ng imiquimod, or PBS as control; BALF was collected 16 hours later. Total cell counts (A) and differential cellular composition (B) for BALF were determined via flow cytometry and Giemsa and May-Grünwald staining cytospin analyses. Differential: White, macrophages; black, neutrophils; gray, eosinophils. Data are expressed as means \pm SEM. ${ }^{*} P<0.05,{ }^{* * * * *} P<0.0001$ versus naïve mice $(\mathbf{A}) .{ }^{* *} P<0.01,{ }^{* * * * *} P<0.001$ versus macrophage numbers in correspondingly stimulated naïve lungs $(\mathbf{B})$ ${ }^{+} P<0.001$ versus neutrophil numbers in correspondingly stimulated naïve lungs $(\mathbf{B}) .{ }^{\sharp} P<0.01,{ }^{\sharp \# P} P<0.001$ versus eosinophil numbers in correspondingly stimulated naïve lungs $(\mathbf{B})$.

\section{Discussion}

Inflammatory responses are characterized as highly dynamic processes. Once local innate immune cells are activated by the inflammatory insult, cytokine and chemokine secretion results in different waves of leukocyte recruitment to the site of inflammation. After elimination of the antigenic threat, inflammation is cleared and the tissue eventually strives to regain steady-state conditions. In the present study, we used a mouse model of eosinophilic asthma in which OVA-alum-sensitized mice are exposed to nebulized OVA, resulting in the infiltration of the bronchoalveolar lumen with eosinophils, CD4 ${ }^{+} \mathrm{T}$-lymphocytes (predominantly Th2 cells), and monocytes. Once allergen exposure was arrested, the bronchial inflammation dampened and eventually cleared. Absolute cell numbers returned to basal levels, and the cellular composition of the alveoli again consisted nearly exclusively of rAMs. Phenotypic analysis of this postinflammation rAM population revealed few differences between naïve and inflammation-experienced rAMs. CD11c and
DEC-205, rAM surface markers not found on other macrophage populations and expressed mainly by dendritic cells and dendritic cell subpopulations, ${ }^{15-20}$ were equally expressed on both pre- and postinflammation rAMs. High intrinsic fluorescence intensities were also found on both rAM populations. However, postinflammation rAMs exhibited higher levels of CD11b, another member of the integrin family that is historically considered to be the canonical macrophage marker, ${ }^{22}$ and of CD115, the receptor for the monocyte/macrophage growth factor M-CSF. ${ }^{4}$

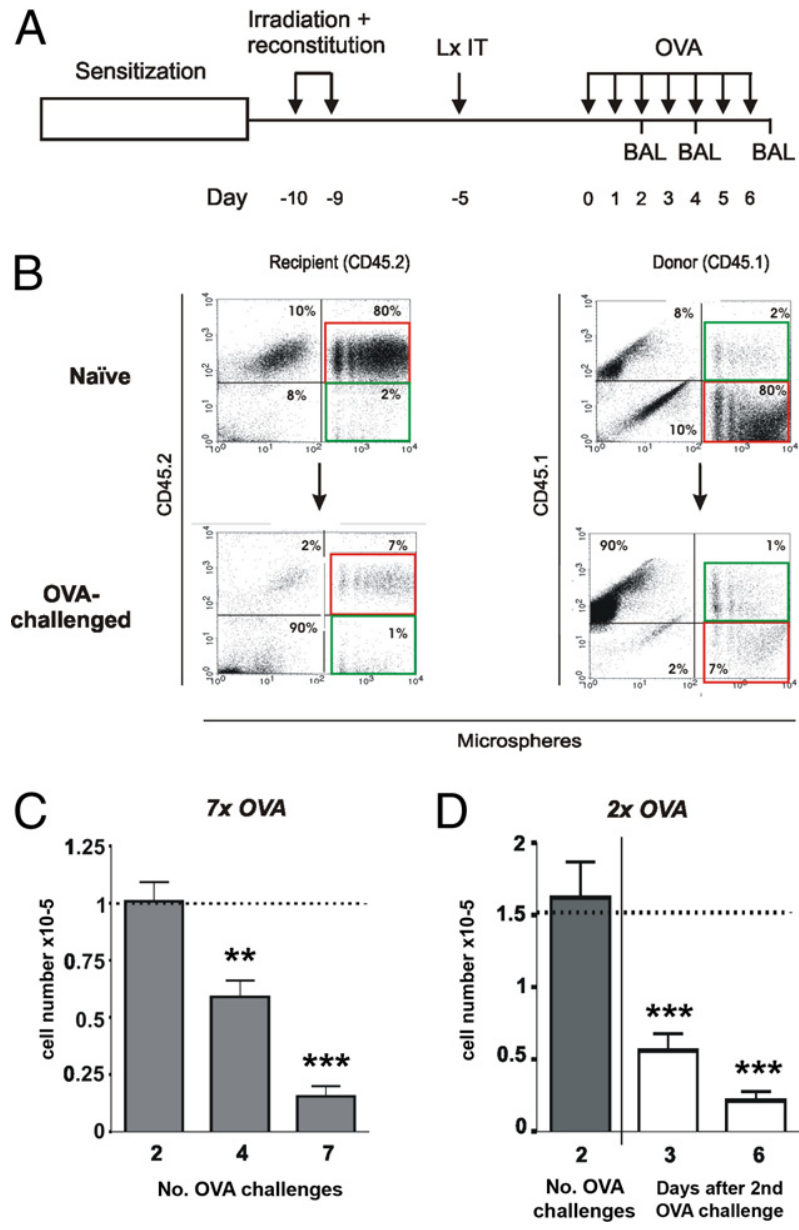

Figure 5. Naïve rAM turnover in response to allergen exposure. A: OVAalum-sensitized wild-type C57BL/6 mice (CD45.2) $(n=7)$ were irradiated and the depleted bone marrow was reconstituted intravenously with CD45.1expressing donor bone marrow cells at days -10 and -9 , respectively. $\mathrm{YG}^{+}$ microspheres were administered intrathecally at day -5 (Lx IT), followed at day 0 by exposure of the mice to OVA aerosol or no challenge (control). B: Naïve rAMs from unchallenged CD 45 chimeric mice were identified by flow cytometry as $\mathrm{CD} 45.2^{+}$microsphere $^{+}$cells (red gate) within the macrophage/granulocyte gate. Elicited AMs were identified as CD $45.1^{+}$microsphere $^{-}$cells (green gate). C: The presence of naive rAMs (microsphere CD $45.2^{+}$cells) in BALF samples collected after two, four, and seven OVA exposures (on days 3,5 , and 8 , respectively) was determined by flow cytometry, as described for B. Average absolute naïve rAM numbers were calculated from the percentages of microsphere ${ }^{+} \mathrm{CD} 45.2^{+}$cells and absolute alveolar cell numbers as determined by flow cytometry, and were compared with the unchallenged control group (broken line) D: In a second set-up, OVA-alum-sensitized CD 45 chimeric mice $(n=7)$ were exposed twice to OVA aerosol. BAL was performed 1,3 , and 6 days after the second OVA exposure. Average absolute naïve rAM numbers were obtained as in C. Dotted line, unchallenged control mice $(\mathbf{C}$ and $\mathbf{D})$. Data are expressed as means \pm SEM and are representative of two independent experiments. ${ }^{* *} P<$ $0.01,{ }^{* * * *} P<0.0001$. 

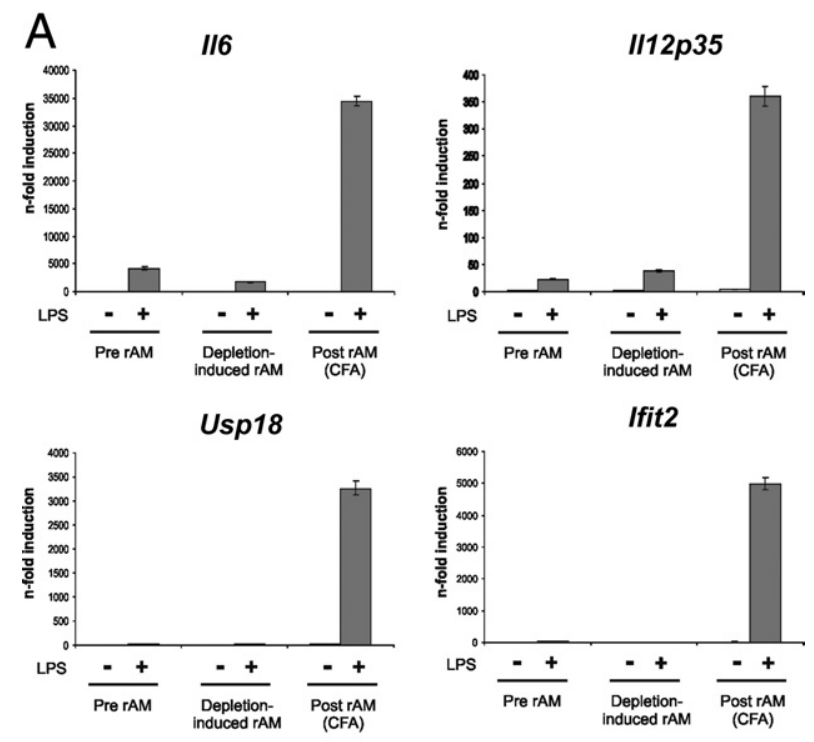

B

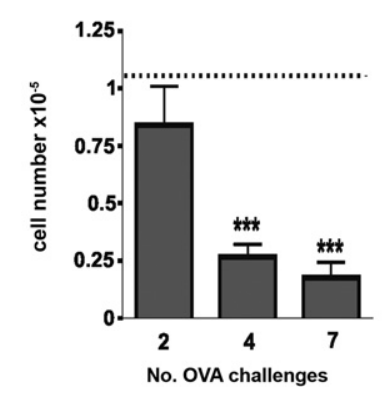

Figure 6. Inflammation-induced rAM turnover is crucial for the development of the postinflammation rAM phenotype. A: Naïve rAMs from C57BL/6 mice were depleted by intrathecal instillation of $30 \%$ clodronate liposomes. OVA-CFA-sensitized C57BL/6 mice were exposed seven times to OVA aerosol. BAL was performed at day 15 after clodronate treatment or instillation of mock liposomes or at day 15 after the last (seventh) OVA challenge $(n=7)$. Mock-treated naïve rAMs, depletion-induced secondary rAMs, and postinflammation rAMs from the noneosinophilic severe refractory asthma model were stimulated ex vivo with $0.1 \mu \mathrm{g} / \mathrm{mL}$ LPS for 6 hours. qPCR analysis was used to determine the mRNA transcript levels of Il6, Il12p35, Usp18, and Ifit2. Data are expressed as means \pm SD from triplicate PCR reactions; $n$-fold induction is relative to unstimulated rAMs from mock-treated mice. B: OVACFA-sensitized wild-type C57BL/6 mice (CD45.2) $(n=7)$ were irradiated, and the depleted bone marrow was reconstituted intravenously with CD45.1expressing donor bone marrow cells at days -10 and -9 , respectively. $\mathrm{YG}^{+}$ microspheres were administered intrathecally at day -5 , followed at day 0 by exposure of the mice to OVA aerosol, or were left unchallenged as control. BALF samples were collected after two, four, and seven OVA exposures. The presence of naïve rAMs (microsphere ${ }^{+} \mathrm{CD} 45.2^{+}$) in the BALF was determined as described above (Figure 5, B and C). Error bars indicate SEM. Dotted line, naïve control. Data are representative of two independent experiments. ${ }^{* * * *} P<0.0001$

Despite postinflammation rAMs being phenotypically nearly indistinguishable from naïve rAMs, the two populations differed dramatically in innate functionality. rAMs readily engulf opsonized and nonopsonized particulate matter. ${ }^{23}$ This high phagocytic capacity is also illustrated by the high uptake of latex microspheres that we observed in cultures of preinflammation rAMs. However, postinflammation rAMs appear to have lost this phagocytic capacity, exhibiting a nearly 10 -fold decrease in latex beads engulfed per cell after 6 hours and a similar decrease in the number of cells having engulfed latex beads. Contrary to this reduced phagocytic capacity, ex vivo TLR stimulation of postinflammation rAMs exhibited a strongly enhanced inflammatory cytokine and chemokine response. Secreted levels of inflammatory mediators such as TNF- $\alpha$, IL-6, IL-12p70, CXCL1, and CXCL2 were increased after LPS and imiquimod stimulation, compared with levels in naïve rAMs. These data indicate a shift from a naïve rAM phenotype exerting phagocytic clearance of inhaled microparticles with a minimal inflammatory reactivity to a postinflammation rAM phenotype possibly responding to a microbial insult by secreting a full range of inflammatory mediators.

Further evidence for a shift toward a less tightly controlled inflammatory phenotype derives from the strikingly different regulation of IFN- $\beta$ expression observed in postinflammation rAMs. Although naïve rAMs possess a functional IFNAR signaling that renders them fully responsive to exogenous type I IFN, the cells characteristically fail to autonomously secrete IFN- $\beta$ after engagement of TLR-3 or TLR-4. ${ }^{26}$ However, when postinflammation rAMs were assayed for this trait they produced significant levels of bioactive IFN- $\beta$ after TLR- 4 and TLR-3 stimulation by LPS and poly $\mathrm{I}: \mathrm{C}$, respectively. TLR-4 is the only TLR family member that uses all four known downstream adaptor molecules (TIRAP, MyD88, TRIF, and TRAM). ${ }^{30}$ Engagement of TLR-4 induces the expression of NF- $\kappa$ B target genes via the MyD88-dependent pathway, whereas the type I IFN response (via IRF-3) is induced via the TRIF-dependent pathway. Triggering of TLR-3, however, engages predominantly TRIF-dependent signaling and expression of type I IFN. ${ }^{30}$ In accord with these reports, in the present study TLR-3 triggering by poly I:C selectively induced a potent IFN- $\beta$ response in postinflammation rAMs, whereas naïve rAMs failed to respond. Finally, triggering exclusively the complementary MyD88-signaling pathway by using the TLR-7 ligand imiquimod further confirmed the strongly increased inflammatory reactivity of postinflammation rAMs, as opposed to the hypoinflammatory reactivity of naïve rAMs. Thus, innate imprinting of rAMs by allergic bronchial inflammation causes a switch from a highly phagocytic, hypoinflammatory to a low phagocytic, hyperinflammatory phenotype that can contribute to the increased total pulmonary response to microbial and viral infectious agents.

A shift in the GM-CSF/M-CSF balance may be implicated in the origin of the differential type I IFN responsiveness of postinflammation rAMs. It has been shown that priming of bone marrow-derived macrophages with M-CSF or GM-CSF affects the cytokine repertoire produced after LPS stimulation. GM-CSF-priming of bone marrow-derived macrophages enhanced the expression of genes induced by the MyD88-dependent pathway (eg, genes for Tnf- $\alpha$, IL-12p40, and IL-23p19), whereas MCSF-primed bone marrow-derived macrophages expressed increased levels of IRF-3-induced and secondary, IFN- $\beta$-induced genes (eg, Ifnb and Col5). ${ }^{31}$ The healthy lung constitutes a GM-CSF rich environment. ${ }^{32}$ Imprinting by GM-CSF of naïve rAMs could therefore be implicated in the origin of the defective IFN- $\beta$ production observed in rAMs. A shift toward M-CSF as a result of the preceding allergic bronchial inflammation could then contribute to the enhanced TRIF-dependent TLR signaling seen in postinflammation rAMs. This proposition is 
further supported by the observed dependence of the postinflammation rAM phenotype on a preceding allergic bronchial inflammation, rather than on the inflammationinduced recruitment of monocytes per se. Thus, in the present study the sterile depletion of naïve rAMs by clodronate liposomes followed by a spontaneous repopulation of the lung with a new population of secondary rAMs did not result in the characteristic inflammatory and IFN- $\beta$ positive phenotype of the secondary rAMs. Nonetheless, an accelerated rAM turnover and replacement by newly elicited mononuclear phagocytes may be part of the mechanism underlying this phenomenon of innate imprinting.

Applying the genetically stable and activation-independent CD45.1 and CD45.2 alloantigen expression system to generate chimeric mice expressing the CD45.1 alloantigen on peripheral blood leukocytes and the CD45.2 alloantigen on rAMs, we observed a rapid clearance of naïve rAMs from the bronchoalveolar lumen starting after four OVA-aerosol challenges and their replacement by newly recruited monocytes/macrophages. Similar findings have been reported using a mouse model for acute respiratory distress syndrome, in which a rapid rAM turnover is induced by a single bolus instillation of $E$. coli LPS. ${ }^{33} \mathrm{~A}$ study of rAM turnover kinetics in mice infected with Streptococcus pneumoniae also revealed a brisk replacement of rAMs by elicited macrophages during the innate phase of the infection. ${ }^{34}$ Thus, an inflammation-driven clearance of rAMs may facilitate their replacement by a novel population of rAMs that have acquired an altered innate response profile as a result of an altered inflammatory cytokine profile, such as a shift in the basal GM-CSF/M-CSF balance. The generic nature of this mechanism of innate imprinting is further illustrated by the similar rAM clearance kinetics and postinflammation rAM functional phenotype observed in a recently established mouse model for Th1- and Th17-biased noneosinophilic severe refractory asthma. ${ }^{29}$ Nevertheless, the nature of the inflammatory response may involve some fine tuning of the basal differentiation of the postinflammation rAMs. Thus, in the eosinophilic asthma model, Th2-associated cytokines such as IL-4 may promote an M2 differentiation state, as suggested by the increased $\operatorname{Arg} 1 / /$ nos ratio we observed in this model.

In conclusion, alveolar macrophages exist in an environment high in antigenic material, of which the majority must be ignored except when the antigen represents an infectious threat. ${ }^{35,36}$ This rAM phenotype provides a basal level of restraint that can be elegantly overridden in the presence of infection. Here we have demonstrated that this tightly controlled cell population rapidly disappears from the alveoli after a brief exposure to allergen and is replaced by a new postinflammation rAM population exhibiting altered innate characteristics. Along with a reduced phagocytic clearance of (inhaled) microparticles and an increased inflammatory TLR signaling, the acquisition of an autonomous IFN- $\beta$ secretion capacity is among the most striking features of this phenomenon of innate imprinting. Until now, most reports on innate imprinting identified TLR desensitization and/or a more restrained inflammatory phenotype as the main features of imprinted macrophages. ${ }^{12,13}$ Here we have demonstrated an oppo- site outcome, namely, an immunologically released rAM phenotype, possibly contributing to the increased sensitivity of the allergic lung toward bacterial and viral infections and renewed exposure to allergen.

\section{Acknowledgments}

We thank Dr. Luc Vakaet (Ghent University Hospital, Ghent, Belgium) for the irradiation of mice, Dr. Anje Cauwels (VIB/Ghent University, Ghent, Belgium) for performing the intravenous injections, and Samuel Noppen for technical support.

\section{References}

1. Barnes PJ: Immunology of asthma and chronic obstructive pulmonary disease. Nat Rev Immunol 2008, 8:183-192

2. Cohn L, Elias JA, Chupp GL: Asthma: mechanisms of disease persistence and progression. Annu Rev Immunol 2004, 22:789-815

3. de Heer HJ, Hammad $H$, Soullie T, Hijdra D, Vos N, Willart MA Hoogsteden HC, Lambrecht BN: Essential role of lung plasmacytoid dendritic cells in preventing asthmatic reactions to harmless inhaled antigen. J Exp Med 2004, 200:89-98

4. Auffray C, Sieweke MH, Geissmann F: Blood monocytes: development, heterogeneity, and relationship with dendritic cells. Annu Rev Immunol 2009, 27:669-692

5. Lambrecht BN: Alveolar macrophage in the driver's seat. Immunity 2006, 24:366-368

6. Ho AS, Moore KW: Interleukin-10 and its receptor. Ther Immunol 1994, 1:173-185

7. Holt PG, Oliver J, Bilyk N, McMenamin C, McMenamin PG, Kraal G Thepen $T$ : Downregulation of the antigen presenting cell function(s) of pulmonary dendritic cells in vivo by resident alveolar macrophages. J Exp Med 1993, 177:397-407

8. Thepen T, Van Rooijen N, Kraal G: Alveolar macrophage elimination in vivo is associated with an increase in pulmonary immune response in mice. J Exp Med 1989, 170:499-509

9. Thepen T, McMenamin C, Girn B, Kraal G, Holt PG: Regulation of IgE production in pre-sensitized animals: in vivo elimination of alveolar macrophages preferentially increases IgE responses to inhaled allergen. Clin Exp Allergy 1992, 22:1107-1114

10. Stumbles PA, Upham JW, Holt PG: Airway dendritic cells: co-ordinators of immunological homeostasis and immunity in the respiratory tract. APMIS 2003, 111:741-755

11. Jakubzick C, Tacke F, Llodra J, van Rooijen N, Randolph GJ: Modulation of dendritic cell trafficking to and from the airways. J Immunol 2006, 176:3578-3584

12. Page KR, Scott AL, Manabe YC: The expanding realm of heterologous immunity: friend or foe? Cell Microbiol 2006, 8:185-196

13. Didierlaurent A, Goulding J, Patel S, Snelgrove R, Low L, Bebien M, Lawrence T, van Rijt LS, Lambrecht BN, Sirard JC, Hussell T: Sustained desensitization to bacterial Toll-like receptor ligands after resolution of respiratory influenza infection. J Exp Med 2008, 205:323-329

14. Kim EY, Battaile JT, Patel AC, You Y, Agapov E, Grayson MH, Benoit LA, Byers DE, Alevy Y, Tucker J, Swanson S, Tidwell R, Tyner JW, Morton JD, Castro M, Polineni D, Patterson GA, Schwendener RA, Allard JD, Peltz G, Holtzman MJ: Persistent activation of an innate immune response translates respiratory viral infection into chronic lung disease. Nat Med 2008, 14:633-640

15. Gonzalez-Juarrero M, Shim TS, Kipnis A, Junqueira-Kipnis AP, Orme IM: Dynamics of macrophage cell populations during murine pulmonary tuberculosis. J Immunol 2003, 171:3128-3135

16. Grundy M, Sentman CL: GFP transgenic mice show dynamics of lung macrophages. Exp Cell Res 2005, 310:409-416

17. Paine R 3rd, Morris SB, Jin H, Wilcoxen SE, Phare SM, Moore BB, Coffey MJ, Toews GB: Impaired functional activity of alveolar macrophages from GM-CSF-deficient mice. Am J Physiol Lung Cell Mol Physiol 2001, 281:L1210-L1218

18. van Rijt LS, Kuipers H, Vos N, Hijdra D, Hoogsteden HC, Lambrecht $\mathrm{BN}$ : A rapid flow cytometric method for determining the cellular 
composition of bronchoalveolar lavage fluid cells in mouse models of asthma. J Immunol Methods 2004, 288:111-121

19. Bilyk N, Holt PG: The surface phenotypic characterization of lung macrophages in C3H/HeJ mice. Immunology 1991, 74:645-651

20. Bosio CM, Dow SW: Francisella tularensis induces aberrant activation of pulmonary dendritic cells. J Immunol 2005, 175:6792-6801

21. Vermaelen K, Pauwels R: Accurate and simple discrimination of mouse pulmonary dendritic cell and macrophage populations by flow cytometry: methodology and new insights. Cytometry A 2004, 61:170-177

22. Guth AM, Janssen WJ, Bosio CM, Crouch EC, Henson PM, Dow SW: Lung environment determines unique phenotype of alveolar macrophages. Am J Physiol Lung Cell Mol Physiol 2009, 296:L936-L946

23. Gordon SB, Read RC: Macrophage defences against respiratory tract infections. Br Med Bull 2002, 61:45-61

24. Varin A, Mukhopadhyay S, Herbein G, Gordon S: Alternative activation of macrophages by IL-4 impairs phagocytosis of pathogens but potentiates microbial-induced signalling and cytokine secretion. Blood 2010, 115:353-362

25. Martinez FO, Helming L, Gordon S: Alternative activation of macrophages: an immunologic functional perspective. Annu Rev Immunol 2009, 27:451-483

26. Punturieri A, Alviani RS, Polak T, Copper P, Sonstein J, Curtis JL: Specific engagement of TLR4 or TLR3 does not lead to IFN-beta-mediated innate signal amplification and STAT1 phosphorylation in resident murine alveolar macrophages. J Immunol 2004, 173:1033-1042

27. Erlandsson L, Blumenthal R, Eloranta ML, Engel H, Alm G, Weiss S, Leanderson T: Interferon-beta is required for interferon-alpha production in mouse fibroblasts. Curr Biol 1998, 8:223-226

28. van Rooijen N, Sanders A, van den Berg TK: Apoptosis of macrophages induced by liposome-mediated intracellular delivery of clodronate and propamidine. J Immunol Methods 1996, 193:93-99
29. Bogaert P, Naessens T, De Koker S, Hennuy B, Hacha J, Smet M, Cataldo D, Di Valentin E, Piette J, Tournoy KG, Grooten J: Inflammatory signatures for eosinophilic vs. neutrophilic allergic pulmonary inflammation reveal critical regulatory checkpoints. Am J Physiol Lung Cell Mol Physiol 2011, 300:L679-L690

30. Kawai T, Akira S: The role of pattern-recognition receptors in innate immunity: update on Toll-like receptors. Nat Immunol 2010, 11:373-384

31. Fleetwood AJ, Dinh H, Cook AD, Hertzog PJ, Hamilton JA: GM-CSFand M-CSF-dependent macrophage phenotypes display differential dependence on type I interferon signaling [Erratum appeared in J Leukoc Biol 2012, 91:347]. J Leukocyte Biol 2009, 86:411-421

32. Gordon S, Taylor PR: Monocyte and macrophage heterogeneity. Nat Rev 2005, 5:953-964

33. Maus UA, Janzen S, Wall G, Srivastava M, Blackwell TS, Christman JW, Seeger W, Welte T, Lohmeyer J: Resident alveolar macrophages are replaced by recruited monocytes in response to endotoxin-induced lung inflammation. Am J Respir Cell Mol Biol 2006, 35:227-235

34. Taut K, Winter C, Briles DE, Paton JC, Christman JW, Maus R, Baumann R, Welte T, Maus UA: Macrophage turnover kinetics in the lungs of mice infected with Streptococcus pneumoniae. Am J Respir Cell Mol Biol 2008, 38:105-113

35. Bingisser RM, Holt PG: Immunomodulating mechanisms in the lower respiratory tract: nitric oxide mediated interactions between alveolar macrophages, epithelial cells, and T-cells. Swiss Med Wkly 2001, 131:171-179

36. Munger JS, Huang X, Kawakatsu H, Griffiths MJ, Dalton SL, Wu J, Pittet JF, Kaminski N, Garat C, Matthay MA, Rifkin DB, Sheppard D: The integrin alpha $v$ beta 6 binds and activates latent TGF beta 1: a mechanism for regulating pulmonary inflammation and fibrosis. Cell 1999, 96:319-328 